\title{
BMJ Open Person-centred transition programme to empower adolescents with congenital heart disease in the transition to adulthood: a study protocol for a hybrid randomised controlled trial (STEPSTONES project)
}

\author{
Mariela Acuña Mora, ${ }^{1,2}$ Carina Sparud-Lundin, ${ }^{1}$ Ewa-Lena Bratt, ${ }^{1,3}$ Philip Moons ${ }^{1,2}$
}

To cite: Acuña Mora M, Sparud-Lundin C, Bratt E-L, et al. Person-centred transition programme to empower adolescents with congenital heart disease in the transition to adulthood: a study protocol for a hybrid randomised controlled trial (STEPSTONES project). BMJ Open 2017;7:e014593. doi:10.1136/bmjopen-2016014593

- Prepublication history for this paper is available online. To view these files please visit the journal online (http://dx.doi.org/10.1136/ bmjopen-2016-014593).

Received 5 October 2016 Revised 19 January 2017 Accepted 23 January 2017

CrossMark

For numbered affiliations see end of article.

Correspondence to Professor Philip Moons; philip.moons@kuleuven.be

\section{ABSTRACT}

Introduction: When a young person grows up, they evolve from an independent child to an empowered adult. If an individual has a chronic condition, this additional burden may hamper adequate development and independence. Transition programmes for young persons with chronic disorders aim to provide the necessary skills for self-management and participation in care. However, strong evidence on the effects of these interventions is lacking. Therefore, as part of the STEPSTONES project (Swedish Transition Effects Project Supporting Teenagers with chrONic mEdical conditionS), we propose a trial to assess the effectiveness of a structured, person-centred transition programme to empower adolescents with congenital heart disease in the transition to adulthood.

Methods/design: STEPSTONES will use a hybrid experimental design in which a randomised controlled trial is embedded in a longitudinal, observational study. It will be conducted in 4 paediatric cardiology centres in Sweden. 2 centres will be allocated to the randomised controlled trial group, assigning patients randomly to the intervention group $(n=63)$ or the comparison group $(n=63)$. The other 2 centres will form the intervention-naïve control group $(n=63)$. The primary outcome is the level of patient empowerment, as measured by the Gothenburg Young Persons Empowerment Scale (GYPES).

Ethics and dissemination: The study has been approved by the Regional Ethical Board of Gothenburg, Sweden. Findings will be reported following the CONSORT statement and disseminated at international conferences and as published papers in peer-reviewed journals.

Trial registration number: NCT02675361; pre-results.

\section{BACKGROUND}

In developed countries, $10-40 \%$ of the adolescent population has a chronic disease with childhood onset. ${ }^{1}$ As a result of improvements

\section{Strengths and limitations of this study}

- This is a multicentre study that uses a novel study design to test the effectiveness of a person-centred transition programme.

- Although several guidelines have recommended the implementation of transition interventions, the evidence of these is still insufficient. The study is expected to fill a gap in current transitional care knowledge.

- Owing to the type of intervention, blinding of participants is not possible.

- In order to have a long follow-up, transition interventions are recommended to start before the age of 16 years, which is not feasible in this study.

in diagnostic techniques and treatments, survival has increased, and $90 \%$ of them now reach adulthood. ${ }^{2}$ Growing up with a chronic condition poses an extra burden on the individual, over and above the challenges typically related to adolescence. Management of the transition from childhood to adulthood and the shift from paediatric to adult care requires special attention from healthcare professionals. The movement to adult care is known as transfer, defined as "an event or series of events through which adolescents and young adults with chronic physical and medical conditions move their care from a pediatric to an adult health care environment". ${ }^{3}$ This transfer should be preceded by a preparatory transitional phase. In healthcare, transition can be defined as the "process by which adolescents and young adults with chronic childhood illnesses are prepared to take charge of their lives and their health in adulthood". ${ }^{3}$ To improve and facilitate this process, transition 
programmes have been developed. ${ }^{4}$ A well-designed transition programme is individually tailored, flexible, and age and developmentally appropriate. ${ }^{5}$ The goal of transition programmes is to support the person's adjustment to a new care culture, to provide the necessary tools for selfcare and to help satisfy medical, psychosocial and educational needs. ${ }^{6}$ Evidence on the effects of transition programmes is scant, the level of existing evidence is low and the effectiveness of these interventions has yet to be determined. ${ }^{7}$ This is due to the limited number of effectiveness studies; methodological issues (eg, contamination of control groups has not been accounted for in prior studies) and a short follow-up period of 12 months maximum. ${ }^{7}$

Patient empowerment is an asset that relates to the adolescents' self-management and participation in care. ${ }^{8}$ Empowered adolescents can interact better with the adult healthcare system, and are more independent and more actively involved in decisions regarding their health. ${ }^{9}{ }^{10}$ However, patient empowerment has not been a formal target in transition programmes so far. To increase the level of empowerment, a person-centred care (PCC) approach is important. ${ }^{11}$ This perspective allows for a partnership between the adolescent and healthcare providers in which the adolescent's views guide the intervention. ${ }^{12}$ Through this approach, it is possible to share helpful resources and information with the adolescents and give them the opportunity to assume an active role in the decision-making process. ${ }^{11}$

Congenital heart disease (CHD) is a typical example of a life-course disease and can therefore be considered a chronic condition. CHD has a prevalence of 9.1 per 1000 newborns and represents $28 \%$ of all congenital defects. ${ }^{13}$ Around $90 \%$ of children born with CHD reach adulthood, ${ }^{2}$ and $\sim 6.2$ in 1000 adults live with this condition. ${ }^{14}$ Although several centres have established a transition programme for individuals with $\mathrm{CHD},{ }^{15}$ only one study on the effectiveness of such a programme has been undertaken so far. That study was conducted in Canada and used a quasi-experimental design to test the effects of a 1-hour intervention led by a nurse. ${ }^{16}$ Significant improvements in the level of knowledge and selfmanagement among patients were observed. ${ }^{16}$ In addition, a comparative and a longitudinal study in Belgium confirmed the effectiveness of patient education. ${ }^{1718}$

To address the gaps in evidence on the effectiveness of transition programmes, we established the STEPSTONES project (Swedish Transition Effects Project Supporting Teenagers with chrONic mEdical conditionS). This project aims at developing and evaluating the effectiveness of transition programmes for young persons with chronic childhood-onset conditions. Although the project will encompass different chronic conditions, it will first study adolescents with CHD.

\section{Objective and hypothesis}

This article describes the protocol for a study to test the effectiveness of a transition programme to empower adolescents with CHD in the transition to adulthood and the transfer to adult care. The main hypothesis is that adolescents with CHD who receive a structured, person-centred transition programme over a 2-year period have a higher patient empowerment score than adolescents receiving usual care.

This study is presented in accordance with the guidelines issued by SPIRIT for reporting of study protocols. ${ }^{19}$

\section{METHODS/DESIGN}

\section{Study design and setting}

A hybrid experimental design will be used, in which a randomised controlled trial (RCT) is embedded in a longitudinal, observational study. This will result in a three-arm design (see figure 1). The study will be conducted in four CHD centres in Sweden. Two of the centres are allocated to the RCT, where participants are randomly assigned to either the intervention group or the comparison group. The other two centres are designated as the control group and represent intervention-naïve centres.

This type of design allows for the investigation of the effectiveness of the transition programme while also taking into account potential contamination of the comparison group in the intervention centres.

\section{Sample size estimation}

Sample size calculation is based on the primary outcome of patient empowerment. The target is an improved patient empowerment score of 5.25 points on a scale from 15 to 75 (ie, 0.5 SD). For two-sided tests with $\alpha=0.05$ and power $=80 \%, 63$ patients are needed in each arm of the RCT. In order to compensate for a potential $10 \%$ dropout rate, we will recruit 70 patients in each arm of the RCT. An additional 70 patients will be recruited in the centres of the control group. Among the four centres, a total of 210 patients will be enrolled (see figure 1).

\section{Participants and recruitment}

Eligible participants are (1) adolescents with CHD, defined as "a gross structural abnormality of the heart or the intrathoracic great vessels that is actually or potentially of functional significance"; ${ }^{20}$ (2) age 16 years; (3) Swedish speaking and (4) literate.

Patients are excluded if they do not have the physical or cognitive capacity to complete the questionnaires, or have acquired heart conditions or heart transplantation. Parents of the adolescents will also be asked to participate. All eligible participants are recruited by a transition coordinator (TC) or a data collection officer (DCO).

\section{Randomisation}

Using a web-based system, a stratified block randomisation with a random variable block size will assign patients of the RCT centres to the intervention group or the comparison group $(1: 1){ }^{21}$ Block randomisation 


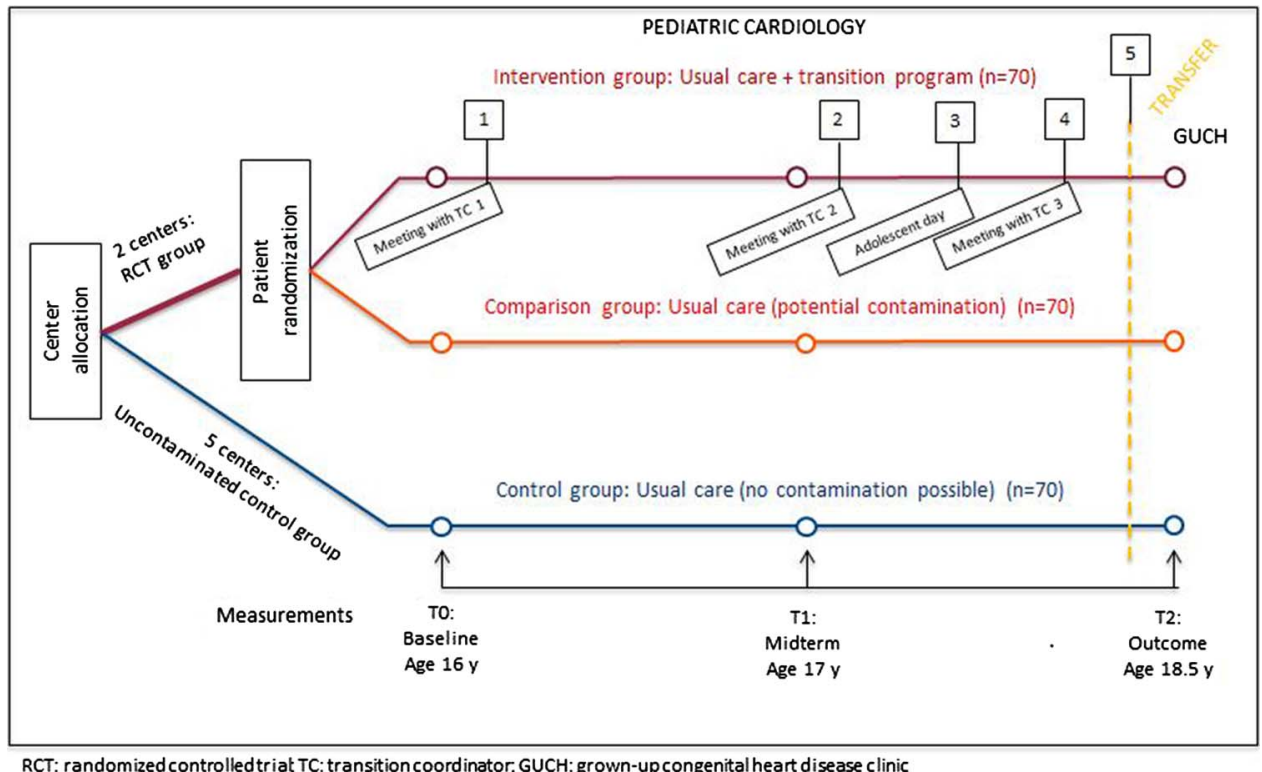

Figure 1 Overview of the study design and implementation steps.

ensures that the TCs in the two centres have a relatively continuous exposure to the intervention over time, which allows them to keep their skills up to date and to ensure the fidelity of the intervention. The fact that the block sizes vary randomly minimises the possibility that the TCs could predict group assignments.

Stratification is made by centre and disease complexity. The stratification for centre ensures that the two centres have an equal number of intervention patients. Stratification by disease complexity (mild; moderate; complex $)^{22}$ is important to avoid over-representation of those people with, for example, complex CHD in one or the other group due to failing randomisation.

Overall, this approach decreases the within-centres variability and reduces the risk for bias and confounding. ${ }^{21}$ The TCs are responsible for accessing the randomisation system in order to determine patient allocation.

\section{Intervention}

Transition programme (intervention group)

This study implements a multicomponent intervention, adapted from a brief transition programme developed by Hilderson et al. ${ }^{23}$ Adaptation of the programme followed the intervention mapping process. ${ }^{24}$ Intervention mapping is a thorough method used to build comprehensive programmes for health promotion and consists of six consecutive steps: (1) assessment of the needs of the target population; (2) defining programme objectives by specifying performance and change objectives; (3) determining theory-based intervention methods and practical strategies; (4) designing and producing the intervention programme; (5) adoption and implementation of the intervention; and (6) developing and executing a plan for evaluation. ${ }^{24}$ Applying the intervention mapping method in this study ensures that the intervention is well-grounded and includes the necessary components to target the programme objectives. A detailed description of each of the steps will be given in a related article.

The transition programme includes eight key components: (1) a TC; (2) a written person-centred transition plan; (3) provision of information and education about the condition, treatment and health behaviours; (4) availability by telephone and email; (5) information about and contact with the Grown-Up Congenital Heart Disease (GUCH) clinic; (6) guidance of parents; (7) meeting with peers; and (8) the actual transfer to the GUCH clinic.

These components are implemented in five steps: (1) a first patient visit with the TC; (2) a second outpatient visit with the TC; (3) information day for adolescents and their parents; (4) a third outpatient visit with the TC; and (5) actual transfer to the GUCH clinic. The TCs are specialised nurses at the outpatient clinic of paediatric cardiology who have received specific training in performing this intervention.

Throughout the intervention, the TCs employ a PCC approach. PCC implies that participants are active agents in their care and decision-making process. ${ }^{12}$ Ekman et $a l^{12}$ proposes that PCC can be implemented in three stages. First, initiating the partnership by allowing the patients to tell their story (patient narratives). This allows for the patients' views to be at the centre of care. Second, developing the partnership through constant communication between the patient and healthcare providers. This is accomplished by sharing experiences and building a common understanding of the care plan. Third, safeguarding the partnership by documenting the decisions made and the preferences of the patients.

The content of the visits is individualised, age and developmentally adapted, and it is documented in the 
individualised transfer plan. The TCs, together with the adolescent, are in charge of determining which topics are important to discuss, in establishing goals related to patient empowerment and in assessing the need for referral to other services.

\section{Usual care (intervention, control and comparison group)}

All adolescents who participate in the study receive usual care, irrespective of whether in the intervention, comparison or control group. Usual care includes visits to an outpatient paediatric cardiology clinic where they meet a nurse and a paediatric cardiologist. Disease complexity determines the frequency of the visits. None of the participating centres have a TC as a part of usual care and there is no documentation of transition plans. It is common practice in Sweden that patients are transferred to adult care around the age of 18 years.

Since usual care can vary across clinics and over time, an assessment of transition-related practices will be carried out in all the centres at the beginning of the intervention and at the end of the study. This assessment will comprise three elements: (1) a structured survey called CHAT (Congenital Heart Adolescent and Transition; on file); (2) interviews with healthcare professionals who are involved in the care for adolescents with CHD in the participating centres and (3) observations of the routine during the clinic visits.

\section{Blinding}

Owing to the nature of the intervention and the study design, it is not possible to blind the participants, TCs or DCOs. However, the TCs and DCOs in charge of carrying out the intervention and data collection are not involved in the preparation of the intervention design or in the statistical analyses.

To avoid cross-contamination between the comparison and the intervention group within the RCT centres, TCs are not involved in the delivery of care of the comparison group and other healthcare professionals in the participating centres will not be informed of the intervention components. DCOs in the control group do not participate in discussions of the intervention.

\section{Intervention fidelity}

To ensure that the transition programme is implemented according to the developed intervention, the TCs are selected based on their experience of working with children and adolescents with CHD. In preparation for this study, they received a specifically designed 4 days training programme, addressing adolescents' health and development; communicating with and interviewing young persons; patient education; PCC; and the detection of and screening for risk behaviours. Training is given by experts in the aforementioned fields and features different pedagogical approaches, for example, group discussions, case assessments and role play. In addition, the TCs were asked to read two textbooks on adolescent health and PCC.
Standard operating procedures (SOPs) for each group of the study were developed in order to ensure consistency throughout the study. These SOPs included: ethical approval; study preparation; study administration; participants recruitment and survey completion; intervention characteristics; usual care; data storage, entry and security; study progress; and publication policy.

Intervention fidelity is monitored as part of the process evaluation. The Medical Research Council (MRC) guidance on process evaluation of complex interventions is applied, ${ }^{25}$ and both quantitative and qualitative methods will be used. In the quantitative assessment, we investigate the acceptability, adherence/fidelity and attrition, as well as potential safety issues. These data are collected using three assessment forms (enrolment/follow-up form, adverse events (AEs) report and intervention implementation form) filled out by the TCs during the intervention period. The qualitative assessment will obtain information about the experiences of and implementation by the TCs and will provide insights into the mechanisms of impact based on the experiences of patients and their parents. The process evaluation will be undertaken as a separate study conducted in parallel with this effectiveness study.

\section{Follow-up period and data collection}

During the 2-year follow-up period, outcome measurements are assessed on three different occasions: T0, baseline when the participants are 16 years old; T1, midterm when the participants are 17 years; and T2, final data collection when the participants have been transferred to adult care and are $~ 18.5-19$ years old (see figure 1).

Participants from the intervention group will be mailed the set of questionnaires 1 month before the outpatient clinic visit. If the questionnaires have not been received by the time of the visit, they will have the opportunity to complete them while waiting for their appointment. Patients from the control and comparison groups may not have a scheduled outpatient visit. Therefore, data collection in these groups is completely undertaken by post when the participants are 16 (T0), 17 (T1) and 18.5 years old (T2).

To minimise non-response in the comparison and control groups, a modified Dillman procedure will be used. ${ }^{26}$ Two weeks after the documents are sent, nonresponders will receive a first personalised reminder in the form of a text message. If after 4 weeks the questionnaires have not been received, a reminder letter and a new set of questionnaires is sent. After 6 weeks, persistent non-responders are contacted by telephone and asked whether they received the questionnaires, if the mailing address is correct, if they want a new set of questionnaires and if they want to continue participating in the study. After 8 weeks, a last reminder is sent to those who agree to participate in the study during the telephone call. 


\section{Outcome measurements}

A background information questionnaire is included, comprising sociodemographic variables such as sex, age, educational level, size of community, place of birth, number of siblings, birth order, living situation, other health conditions and medication (obtained from selfreport and medical file).

Standardised questionnaires are used to assess primary and secondary outcomes. Table 1 presents an overview of the characteristics and psychometric properties of the questionnaires used.

\section{Primary outcome measures}

The primary outcome is patient empowerment, defined as "an enabling process or outcome arising from communication with the healthcare professional and a mutual sharing of resources over information relating to illness, which enhances the patient's feelings of control, self-efficacy, coping abilities and ability to achieve change over their condition". 9 Empowerment includes five dimensions: (1) identity; (2) knowledge and understanding; (3) personal control; (4) decision making; and (5) enabling others. ${ }^{9}$

Since no questionnaire to measure the level of empowerment in young persons with chronic conditions exists, we developed the Gothenburg Young Persons Empowerment Scale-Congenital Heart Disease module (GYPES-CHD). The scale has 15 items, 3 items for each of the aforementioned dimensions. Each item is answered on a five-point Likert scale: strongly disagree; disagree; neither agree nor disagree; agree and strongly agree. A total score is calculated by summing the item scores. The total score ranges from 15 to 75 points, with a higher score reflecting a higher level of patient empowerment.

Face validity was confirmed after cognitive interviews with six young persons with CHD. The psychometric properties of this scale were examined in a crosssectional study in 205 young persons with CHD. Internal consistency of the items was confirmed with a Cronbach's $\alpha$ of 0.821 . No floor or ceiling effect in scoring was observed. A complete description of the psychometric evaluation of GYPES will be provided in another article.

\section{Secondary outcomes measures}

We will measure six secondary outcomes in the adolescents: transition readiness, health behaviours, knowledge about CHD, healthcare usage, quality of life and patientreported health. In the parents, we will measure transition readiness (proxy) and parental uncertainty towards transition.

\section{Transition readiness}

Transition readiness is defined as the "adolescent's readiness to assume complete responsibility for their healthcare and their readiness to transfer to adult medical care". ${ }^{27}$ Transition readiness is evaluated using the Readiness for Transition Questionnaire (RTQ), adolescent and parent versions. These questionnaires examine two aspects. First, the overall transition readiness is assessed, using two items ranging from 1 to 4 . The sum of these two items results in a score ranging from 2 to 8. Second, the frequency of adolescent responsibility and parental involvement are reported in 10 different health behaviours, on a five-point Likert scale. Each variable results in a total score ranging from 10 to 40 , and higher scores indicate higher adolescent responsibility or parental involvement, respectively.

\section{Knowledge about CHD}

Adolescents' knowledge about their CHD is assessed with the Knowledge Scale for adults with Congenital Malformed Hearts (KnoCoMH) ${ }^{28}$ This instrument evaluates the level of knowledge within four domains: disease and its treatment; complications prevention; physical activity; and reproductive issues. ${ }^{28}$ The questionnaire includes 19 items and scores are calculated by dichotomising answers as correct or incorrect. Access to the medical files is needed in order to confirm the answers in some of the items. For participants for whom medical treatment or endocarditis prophylaxis is not recommended, the domains are excluded.

\section{Health behaviours}

Health behaviours are measured with the Health Behavior Scale-CHD. Health behaviours are 'activities a person undertakes to maintain or improve health and prevent diseases'. ${ }^{30}$ This scale assesses alcohol consumption, tobacco use, dental care and physical activity. The scale has 15 items that help to calculate three summary risk scores: substance use (score 0-100), dental hygiene (score 0-100) and total health risk score (score 0-100). Higher risk scores represent unhealthier behaviours.

\section{Patient-reported health}

Patient-reported health is assessed by the generic and cardiac modules of the Pediatric Quality of Life inventory 4.0 (PedsQL 4.0), as well as the EuroQoL 5 dimensions 3 levels (EQ-5D-3L). The PedsQL 4.0 provides information on health status in relation to psychosocial and physical health. ${ }^{32}$ The generic module consists of 23 items that cover four dimensions: physical; emotional; social functioning and school functioning. Items are answered by using a five-point Likert scale: never, almost never, sometimes, often and always. For each dimension, a scale score can be calculated, which ranges from 0 to 100. Further, a physical health summary score, a psychosocial health summary score and a total score can be calculated.

The cardiac module contains 27 items that cover six dimensions: symptoms; treatment; perceived physical appearance; treatment anxiety; cognitive problems and communication. Each item is answered using a five-point Likert scale (never, almost never, sometimes, often and always). As in the generic version, scores are 
Table 1 Psychometric properties of the questionnaires used in STEPSTONES-ConHD

\begin{tabular}{|c|c|c|c|c|c|c|c|}
\hline Variable & Subject & Measurements & Items & Validity & Reliability & Responsiveness & Interpretation \\
\hline \multicolumn{8}{|l|}{ Primary outcome } \\
\hline $\begin{array}{l}\text { Patient } \\
\text { empowerment }\end{array}$ & $A D$ & GYPES & 15 & $\begin{array}{l}\text { Construct validity supported an } \\
\text { adequate model fit of a five factor } \\
\text { solution (df: } 80 ; \chi^{2}: 154-948 \text {, } \\
\text { p<0.0001; CFI: } 0.916 \text {; RMSEA: } 0.068 \text {; } \\
\text { SRMR: } 0.069 \text { ) (data on file) } \\
\text { Face validity confirmed in young } \\
\text { persons with chronic conditions (data } \\
\text { on file) }\end{array}$ & $\begin{array}{l}\text { Internal consistency } \\
\text { confirmed in young } \\
\text { persons with CHD, } \\
\alpha=0.819 \text { (data on file) }\end{array}$ & $\begin{array}{l}\text { Floor scores: } 0 \% \\
\text { ceiling scores: } 1.5 \% \\
\text { (data on file) }\end{array}$ & $\begin{array}{l}\text { Score from } 15 \text { to } \\
75 . \text { Higher score } \\
\text { reflects a higher } \\
\text { level of patient } \\
\text { empowerment }\end{array}$ \\
\hline \multicolumn{8}{|c|}{ Secondary outcomes } \\
\hline \multirow[t]{2}{*}{$\begin{array}{l}\text { Transition } \\
\text { readiness }\end{array}$} & $A D$ & $\begin{array}{l}\text { RTQ adolescent } \\
\text { version }\end{array}$ & 26 & $\begin{array}{l}\text { Validity based on relationships with } \\
\text { other variables confirmed in young } \\
\text { persons with kidney transplant, }{ }^{27} \text { p.91- } \\
92\end{array}$ & $\begin{array}{l}\text { Internal consistency } \\
\text { confirmed in young } \\
\text { persons with kidney } \\
\text { transplant; } \alpha \text { values } \\
\text { over } 0.70^{27}\end{array}$ & NR & $\begin{array}{l}\text { Scores from } 10 \text { to } \\
40 . \text { Higher scores } \\
\text { denote increased } \\
\text { adolescent or } \\
\text { caregiver } \\
\text { responsibility. }\end{array}$ \\
\hline & PA & $\begin{array}{l}\text { RTQ parent } \\
\text { version }\end{array}$ & & $\begin{array}{l}\text { Construct validity reported for parents } \\
\text { of young persons with kidney } \\
\text { transplant, }{ }^{27} \text { p.91-92 }\end{array}$ & $\begin{array}{l}\text { Reliability reported for } \\
\text { parents of young } \\
\text { persons with kidney } \\
\text { transplant; } \alpha \text { values } \\
\text { over } 0.70,{ }^{27} \text { p.90 }\end{array}$ & NR & \\
\hline $\begin{array}{l}\text { Knowledge on } \\
\text { ConHD }\end{array}$ & $A D$ & KnoCoMH & 19 & $\begin{array}{l}\text { Validity was confirmed in adults with } \\
\mathrm{CHD} \text { in relation to discrimination ability, } \\
\text { the relationship of the items and the } \\
\text { construct of interest, }{ }^{28} \text { p.232-234 } \\
\text { KnoCoMH is based on the Leuven } \\
\text { Knowledge Scale which has been } \\
\text { validated in young persons with } \\
\mathrm{CHD},{ }^{29} \text { p. } 79-80\end{array}$ & $\begin{array}{l}\text { Internal consistency } \\
\text { and test-retest reliability } \\
\text { confirmed, }{ }^{28} \text { p.232-234 }\end{array}$ & NR & $\begin{array}{l}\text { Scores are } \\
\text { calculated by } \\
\text { dichotomising the } \\
\text { answers (correct/ } \\
\text { incorrect) for each } \\
\text { domain }\end{array}$ \\
\hline $\begin{array}{l}\text { Health } \\
\text { behaviours }\end{array}$ & $A D$ & HBS-CHD & $15^{\star}$ & $\begin{array}{l}\text { Item content validity, scale content } \\
\text { validity index and validity based on } \\
\text { relationships to other variables } \\
\text { confirmed in adolescents with } \mathrm{CHD},{ }^{30} \\
\text { p.548 }\end{array}$ & Stability not confirmed 29 & $\begin{array}{l}\text { Confirmed in } \\
\text { adolescents with CHD } \\
\text { by Guyatt's } \\
\text { Responsiveness } \\
\text { Index, }{ }^{30} \text { p.551 }\end{array}$ & $\begin{array}{l}\text { Substance use, } \\
\text { dental hygiene and } \\
\text { total health risk } \\
\text { score from } 0 \text { to } \\
100 \text {. Physical } \\
\text { exercise score } \\
\text { from } 0 \text { to } \infty\end{array}$ \\
\hline $\begin{array}{l}\text { Patient-reported } \\
\text { health }\end{array}$ & $A D$ & $\begin{array}{l}\text { PedsQL generic } \\
\text { module }\end{array}$ & 23 & $\begin{array}{l}\text { Convergent validity, measurement } \\
\text { invariance and factor structure } \\
\text { confirmed in paediatric populations, } \\
{ }^{31} \text { p.336-337 } \\
{ }^{32} \text { p.1507-1510 }\end{array}$ & $\begin{array}{l}\text { Test-retest reliability } \\
\text { and internal consistency } \\
\text { show } \alpha \text { values over } \\
0.70 \text { in paediatric } \\
\text { populations, }{ }^{32} \text { p. } 1507 \\
{ }^{31} \text { p.333-335 }\end{array}$ & $\begin{array}{l}\text { Minimal clinically } \\
\text { important difference } \\
\text { reported for young } \\
\text { people with CHD was } \\
6.0 \text { for the total } \\
\text { score. }^{33}\end{array}$ & $\begin{array}{l}\text { Scores from } 0 \text { to } \\
100 . \text { Higher scores } \\
\text { indicate a better } \\
\text { perceived health } \\
\text { status. }\end{array}$ \\
\hline
\end{tabular}


Table 1 Continued

\begin{tabular}{|c|c|c|c|c|c|c|c|}
\hline Variable & Subject & Measurements & Items & Validity & Reliability & Responsiveness & Interpretation \\
\hline & & $\begin{array}{l}\text { PedsQL cardiac } \\
\text { module }\end{array}$ & 27 & $\begin{array}{l}\text { Convergent validity was confirmed, }{ }^{34} \\
\text { p.215 }\end{array}$ & $\begin{array}{l}\text { Internal consistency for } \\
\text { majority of scales } \\
\text { exceeded } \alpha \text { values of } \\
0.70,{ }^{34} \text { p.214 }\end{array}$ & $\begin{array}{l}\text { Minimal clinically } \\
\text { important differences } \\
\text { reported for young } \\
\text { people with CHD } \\
\text { ranged from } 7.6 \text { for } \\
\text { symptoms to } 12.6 \text { for } \\
\text { communication. }{ }^{3}\end{array}$ & $\begin{array}{l}\text { Scores from } 0 \text { to } \\
100 \text {. Higher scores } \\
\text { indicate lower } \\
\text { problems. }\end{array}$ \\
\hline & & EQ-5D-3L & 5 & $\begin{array}{l}\text { Convergent and discriminative validity } \\
\text { confirmed in patients with } \\
\text { cardiovascular disease }{ }^{35} \text { p.6 }\end{array}$ & $\begin{array}{l}\text { Test-retest reliability } \\
\text { confirmed in } \\
\text { cardiovascular } \\
\text { patients, }{ }^{35} \text { p. } 6\end{array}$ & $\begin{array}{l}\text { Confirmed in patients } \\
\text { with cardiovascular } \\
\text { disease },^{35} \text { p. } 6\end{array}$ & $\begin{array}{l}\text { Scores from } 1 \text { (no } \\
\text { problems) to } 3 \\
\text { (extreme } \\
\text { problems) on five } \\
\text { dimensions } \\
\text { Scores from 0 } \\
\text { (worst imaginable } \\
\text { health state) to } \\
100 \text { (best } \\
\text { imaginable health } \\
\text { state) }\end{array}$ \\
\hline Quality of life & $A D$ & LAS & 1 & $\begin{array}{l}\text { Validity based on relationship with } \\
\text { Satisfaction with Life Scale found to be } \\
\text { highly correlated }(\rho=0.52) \text {. Test content } \\
\text { confirmed in adults with CHD ( } 100 \% \text { of } \\
\text { patients understood the wording and } \\
\text { format) }^{36} \\
\text { Validity has been evaluated in }^{\text {adolescents with CHD. }}{ }^{37} 38\end{array}$ & $\begin{array}{l}\text { Test-retest reliability } \\
\text { confirmed in adults with } \\
\mathrm{CHD}(\mathrm{ICC}=0.65),{ }^{36}\end{array}$ & $\begin{array}{l}\text { Confirmed in adults } \\
\text { with } \mathrm{CHD} \text { (floor } \\
\text { scores }=0 \% \text {, ceiling } \\
\text { scores }=2.7 \%)^{36}\end{array}$ & $\begin{array}{l}\text { Score is from } 100 \\
\text { (best imaginable } \\
\text { health status) to } 0 \\
\text { (worst imaginable } \\
\text { health status). }\end{array}$ \\
\hline $\begin{array}{l}\text { Healthcare } \\
\text { usage }\end{array}$ & $A D$ & $\begin{array}{l}\text { Healthcare } \\
\text { usage } \\
\text { questionnaire }\end{array}$ & 11 & NA & NA & NA & NA \\
\hline $\begin{array}{l}\text { Parental } \\
\text { uncertainty } \\
\text { towards transfer } \\
\text { to adult care }\end{array}$ & PA & LAS & 1 & $\begin{array}{l}\text { Face validity confirmed in parents of } \\
\text { young persons with chronic conditions } \\
\text { (data on file). } \\
\text { Test content confirmed in parents of } \\
\text { young persons with CHD ( } 100 \% \text { of } \\
\text { patients understood the wording and } \\
\text { format). }\end{array}$ & NR & $\begin{array}{l}\text { Floor scores: } 7.8 \% \\
\text { ceiling scores: } 4.8 \% \\
\text { (data on file) }\end{array}$ & $\begin{array}{l}\text { Score is from } 0 \\
\text { (very uncertain } \\
\text { about the transfer } \\
\text { to adult care) to } \\
100 \text { (not uncertain } \\
\text { about the transfer } \\
\text { of care). }\end{array}$ \\
\hline
\end{tabular}

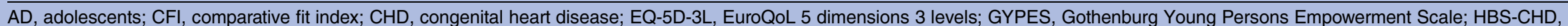
Health Behavior Scale-Congenital Heart Disease; ICC, intra-class correlation; KnoCoMH, Knowledge Scale for Adults with Congenitally Malformed Hearts; LAS, linear analogue scale;

NA, not applicable; NR, not reported; PA, parents; PedsQL, Pediatric Quality of Life Inventory 4.0; RMSEA, root mean square error of approximation; RTQ, Readiness for Transition

Questionnaire; SRMR, standardised root mean square residual; STEPSTONES, Swedish Transition Effects Project Supporting Teenagers with chrONic mEdical conditionS. 
transformed on a scale from 0 to 100 , and then a mean subscale core is calculated. ${ }^{34}$

The EQ-5D-3L comprises a descriptive system and a visual analogue scale (VAS). The descriptive system has five dimensions (mobility, self-care, usual activities, pain/discomfort and anxiety/depression) with three response categories per dimension. ${ }^{39}$ The participants are asked to indicate which statement is most suitable in relation to their health status. The VAS measures the participants' self-rated health on a scale with the end points 'best imaginable health possible' $(=100)$ and 'worst imaginable health state' $(=0)$. The descriptive system allows for the determination of a five-digit health state that combines the level of perceived problems in each dimension (level 1: indicating no problem, level 2: indicating some problems and level 3: indicating extreme problems). Information obtained from the descriptive system can be converted to a single summary index by assigning values to each level of every dimension.

\section{Quality of life}

Quality of life is defined as "the overall degree of life satisfaction that is positively or negatively influenced by individuals' perception of certain aspects of life important to them, including matters both related and unrelated to health". ${ }^{40}$ This variable is measured using a $10 \mathrm{~cm}$ linear analogue scale (LAS), ${ }^{36}$ where the participants indicate in the scale how they judge their current quality of life. The scale looks similar to the VAS of the EQ-5D, but has the end points 'best imaginable quality of life' $(=100)$ and 'worst imaginable quality of life' $(=0)$.

\section{Healthcare usage}

Healthcare usage within the preceding 6 months is measured in relation to their CHD and other possible conditions. The measurement consists of six dimensions: hospital stay; healthcare contact; specialised care; emergency care; school absence and follow-up unit after transfer to adult care. This assessment was developed to be used in the APPROACH-IS project. ${ }^{41}$ After the transfer to adult care, we will assess the proportion of patients who are in follow-up in a GUCH unit, both in the intervention and control arms.

\section{Parental uncertainty}

Parental uncertainty is understood as the degree of certainty/uncertainty the parents have in relation to their children being transferred to adult care. This outcome is measured through LAS with the end points 'extremely uncertain' $(=100)$ and 'not uncertain at all' $(=0)$. This LAS has been developed for the purpose of the present study.

In addition to the primary and secondary end points, we will calculate a composite end point. We will do so because the transition programme is a complex intervention that may have varying effects on different outcomes. Prior studies on the impact of transition programmes showed that the intervention has impacted on transition readiness, ${ }^{16}$ patient knowledge ${ }^{16}$ quality of life $^{42}$ and psychosocial health. ${ }^{42}$ Therefore, each patient will be categorised as improved or not. Patients will be classified as improved if they have an improvement in empowerment, transition readiness, knowledge, quality of life or psychosocial health of 0.5 SDs compared with their baseline measurement.

\section{Data management}

Data are entered into a web-based system by the TC and the DCO. Those with access to the system are the TC, DCO, project coordinator and principal investigator, each of whom has a personal login. All data are checked for completeness and the quality of the data is monitored at least twice a year by the members of the research group. This process is carried out by verifying records with the raw data and checking frequency distributions to identify responses outside of the given possibilities.

\section{Statistical analyses plan}

The primary analyses will be performed following the intention-to-treat principle which helps to avoid biases in the comparisons between groups. The primary end point (level of empowerment) will be analysed using Fisher's non-parametric permutation test unadjusted between the two groups. Complementary between-group analyses will be made using analysis of covariance adjusted for baseline variables that differ significantly between groups and for each centre.

All analyses will be predefined in a comprehensive statistical analysis plan before data lock. The level of significance will be at $\mathrm{p} \leq 0.05$. Two-sided tests will be used.

\section{Trial duration}

Patient recruitment began in July 2016 and is planned to finish in December 2017. Once recruited, patients are followed up until they are transferred to GUCH. Data collection is expected to be finished by December 2019 . After the data set is locked, analysis and dissemination of results will begin.

\section{Ethical considerations}

Before inclusion in the study, the TCs and DCOs will seek written informed consent from the adolescents and their parents after they have received written and oral information about the study. Participants have the right to withdraw at any point throughout the study. The principles established in the Declaration of Helsinki will be followed during the entire duration of the study. ${ }^{43}$

To ensure confidentiality, personal data are coded. Participant and study-related information is stored in locked cabinets and digital documents are passwordprotected.

\section{Follow-up on AEs}

All AEs must be reported to the principal investigator and project coordinator within a week. In case of a 
serious $\mathrm{AE}$, the report must be made immediately. Appropriate measures will be undertaken in every $\mathrm{AE}$ in order to ensure patient safety.

\section{Dissemination}

The results of this study will be submitted to peerreviewed journals for publication following the CONSORT statement. ${ }^{44}$ Abstracts for poster or oral presentations will be submitted to international conferences. Authorship is granted to a collaborator from every participating centre and members of the research group fulfilling the criteria of authorship. ${ }^{45}{ }^{46}$ If possible, we will include 'on behalf of STEPSTONES-CHD' in the list of authors. After completion of the study, the data set will be available on formal request.

\section{DISCUSSION}

Previous studies have tried to determine the effectiveness of transition programmes. However, owing to several methodological limitations, the true effect of these interventions is still uncertain. ${ }^{7}$ Therefore, we will conduct a study that assesses the effectiveness of a structured, person-centred transition programme to empower adolescents with CHD in the transition to adulthood. Through the implementation of the proposed transition programme, we expect an increase in the level of empowerment. A high level of empowerment can help the adolescent navigate through the adult care system, participate in care planning, and increase selfmanagement and decision-making skills. ${ }^{8}$

The intervention in this trial was designed and initially evaluated by Hilderson $e t a l^{23} 42$ in Belgium. Therefore, it was important to determine if the intervention components were suitable in the Swedish setting. To accomplish this, five preparatory studies were carried out (four qualitative, one quantitative study). The qualitative studies included individual and focus group interviews with young persons with CHD and their parents. ${ }^{47}{ }^{48}$ These studies provided an overview of the current transition practices in Sweden and the needs of this population before the transfer to adult care. The quantitative study was a cross-sectional study including 205 young persons with CHD, and served as a pilot-test for our primary outcome measurement. Furthermore, the intervention was discussed with a panel of international experts with experience in transition research in adolescents with chronic conditions and patient representatives.

The preparatory studies and the discussion with the panel of international experts improved the quality, feasibility and acceptability of the intervention. This interdisciplinary and comprehensive approach increases the chance of producing a high-quality intervention with positive results. ${ }^{49}$

This study has several strengths, because we explicitly take limitations of prior studies into account. First, we employ an RCT which is the gold standard for the assessment of the effectiveness of interventions and provides the highest level of evidence. The stratified block randomisation that we undertake ensures that the intervention and comparison groups are balanced and that potential confounding factors, such as complexity of the disease or centre characteristics, are equally distributed and therefore minimise the risk of biasing the results.

Second, the proposed hybrid experimental design allows us to account for potential contamination of the control group. Indeed, the three arms allow performance of direct comparison between the intervention and comparison group while enabling a comparison of findings with those obtained in intervention-naive centres.

Third, patient empowerment is used as the primary outcome. Empowerment is argued to be important for the development and application of skills to promote behavioural changes in the adolescents. ${ }^{10}$ Admittedly, empowerment can be seen both as a process and an outcome. ${ }^{10}$ However, being an intermediate outcome, empowerment can be argued to be proximal to the intervention, whereas continuing cardiac care, reduced morbidity or mortality are probably the ultimate, but more distal outcomes.

Fourth, the PCC approach that is used throughout the transition programme is an important asset in the process of empowering patients. ${ }^{11}$ Providing transitional care from a person-centred perspective is novel and has not been evaluated before in this age group.

Fifth, we follow-up the patients for more than 2 years. While doing so, we substantially expand the follow-up period of 12 months as used in prior studies. ${ }^{7}$

Sixth, since usual care may vary across centres and over time, we carry out a formal usual care assessment. Such a usual care assessment is important to draw firm conclusions, and yet this is seldom performed in clinical trials. $^{50}$

Irrespective of these strengths, we anticipate some limitations as well. First, we are not able to blind the participants or the healthcare team that will perform the intervention. To minimise a potential impact, neither the therapeutic team nor the TCs participate in the data analysis. Second, there is a possibility of a relatively long period of recruitment for participants which can require extending the trial period and delaying data analysis. Third, transition interventions have been advised to start early on, for example, between 10 and 12 years of age, and have a long follow-up. ${ }^{51}$ Furthermore, a long follow-up allows continuity of care to be measured in this population and provides insight into the effects of the transition programme over this parameter. Although we recognise the importance of starting early, it would have jeopardised the feasibility of the study, because it would have taken almost a decade to finish recruitment and follow-up until the age of 18 years. Nonetheless, it could be possible to later on assess loss of follow-up in a separate study. 


\section{CONCLUSION}

This article described the study protocol for a study to assess the effectiveness of a structured, person-centred transition programme for young persons with CHD. This study accounts for limitations of previous research and addresses an existing gap in the evidence of transitional care. It is hoped that the findings will provide strong evidence of the effectiveness of this intervention in young people with CHD. If successful, this transition programme could potentially be implemented as part of routine care provided to young persons with CHD, and transfer to other chronic conditions can be explored.

\section{Trial status}

Status of the trial at the time of submission: patient recruitment.

\section{Author affiliations}

${ }^{1}$ Institute of Health and Care Sciences, University of Gothenburg, Gothenburg, Sweden

${ }^{2}$ Department of Public Health and Primary Care, KU Leuven, Leuven, Belgium ${ }^{3}$ Department of Pediatric Cardiology, The Queen Silvia Children's Hospital, Gothenburg, Sweden

Acknowledgements The authors would like to acknowledge the collaboration from the members of the panel of international experts and of the Advisory Board.

Funding This study is supported by research grants from the Swedish HeartLung Foundation (grant 20150535); Swedish Research Council for Health, Working Life and Welfare-FORTE (grant STYA-2015/0003); Swedish Children Heart Association; Swedish Research Council (grant 2015-02503); and the Institute of Health and Care Sciences of the University of Gothenburg.

Competing interests None declared.

Ethics approval The study was approved by the Regional Ethical Board of Gothenburg, Sweden (No.931-15).

Provenance and peer review Not commissioned; externally peer reviewed.

Data sharing statement Preliminary data on the psychometric properties of the GYPES and the LAS for parental uncertainty are on file. They will be integrated in a separate article on the psychometric evaluation of the instruments, which will be available in some months.

Open Access This is an Open Access article distributed in accordance with the terms of the Creative Commons Attribution (CC BY 4.0) license, which permits others to distribute, remix, adapt and build upon this work, for commercial use, provided the original work is properly cited. See: http:// creativecommons.org/licenses/by/4.0/

\section{REFERENCES}

1. Kennedy A, Sloman F, Douglass JA, et al. Young people with chronic illness: the approach to transition. Intern Med $J$ 2007;37:555-60.

2. Moons $P$, Bovijn $L$, Budts $W$, et al. Temporal trends in survival to adulthood among patients born with congenital heart disease from 1970 to 1992 in Belgium. Circulation 2010;122:2264-72.

3. Meadows A, Bosco V, Tong E, et al. Transition and transfer from pediatric to adult care of young adults with complex congenital heart disease. Curr Cardiol Rep 2009;11:291-7.

4. American Academy of Pediatrics. A consensus statement on health care transitions for young adults with special health care needs. Pediatrics 2002;110(Pt 2):1304-6.

5. Warnes CA, Williams RG, Bashore TM, et al. ACC/AHA 2008 guidelines for the management of adults with congenital heart disease: a report of the American College of Cardiology/American Heart Association Task Force on practice guidelines (writing committee to develop guidelines on the management of adults with congenital heart disease): developed in collaboration with the American Society of Echocardiography, Heart Rhythm Society, International Society for Adult Congenital Heart Disease, Society for Cardiovascular Angiography and Interventions, and Society of Thoracic Surgeons. Circulation 2008;118:E714-833.

6. Crowley $\mathrm{R}$, Wolfe I, Lock $\mathrm{K}$, et al. Improving the transition between paediatric and adult healthcare: a systematic review. Arch Dis Child 2011;96:548-53.

7. Campbell F, Biggs K, Aldiss SK, et al. Transition of care for adolescents from paediatric services to adult health services. Cochrane Database Syst Rev 2016;4:CD009794.

8. Bravo P, Edwards A, Barr PJ, et al. Conceptualising patient empowerment: a mixed methods study. BMC Health Serv Res 2015;15:252.

9. Small N, Bower P, Chew-Graham CA, et al. Patient empowerment in long-term conditions: development and preliminary testing of a new measure. BMC Health Serv Res 2013;13:263.

10. Anderson RM, Funnell MM. Patient empowerment: myths and misconceptions. Patient Educ Couns 2010;79:277-82.

11. Holmström I, Roing M. The relation between patient-centeredness and patient empowerment: a discussion on concepts. Patient Educ Couns 2010;79:167-72.

12. Ekman I, Swedberg K, Taft C, et al. Person-centered care-ready for prime time. Eur J Cardiovasc Nurs 2011;10:248-51.

13. Dolk H, Loane M, Garne $\mathrm{E}$, et al. Congenital heart defects in Europe: prevalence and perinatal mortality, 2000 to 2005. Circulation 2011;123:841-9.

14. Marelli AJ, Ionescu-Ittu R, Mackie AS, et al. Lifetime prevalence of congenital heart disease in the general population from 2000 to 2010. Circulation 2014;130:749-56.

15. Hilderson D, Saidi AS, Van Deyk K, et al. Attitude toward and current practice of transfer and transition of adolescents with congenital heart disease in the United States of America and Europe. Pediatr Cardiol 2009;30:786-93.

16. Mackie AS, Islam S, Magill-Evans J, et al. Healthcare transition for youth with heart disease: a clinical trial. Heart 2014;100:1113-18.

17. Goossens E, Fieuws S, Van Deyk K, et al. Effectiveness of structured education on knowledge and health behaviors in patients with congenital heart disease. J Pediatr 2015;166: 1370-6. e1.

18. Goossens E, Van Deyk K, Zupancic N, et al. Effectiveness of structured patient education on the knowledge level of adolescents and adults with congenital heart disease. Eur J Cardiovasc Nurs 2014;13:63-70.

19. Chan AW, Tetzlaff JM, Altman DG, et al. SPIRIT 2013 statement: defining standard protocol items for clinical trials. Ann Intern Med 2013:158:200-7.

20. Mitchell SC, Korones SB, Berendes HW. Congenital heart disease in 56,109 births. Incidence and natural history. Circulation 1971;43:323-32.

21. Efird J. Blocked randomization with randomly selected block sizes. Int J Environ Res Public Health 2011;8:15-20.

22. Warnes CA, Liberthson R, Danielson GK, et al. Task force 1: the changing profile of congenital heart disease in adult life. J Am Coll Cardiol 2001;37:1170-5.

23. Hilderson D, Westhovens $\mathrm{R}$, Wouters $\mathrm{C}$, et al. Rationale, design and baseline data of a mixed methods study examining the clinical impact of a brief transition programme for young people with juvenile idiopathic arthritis: the DON'T RETARD project. BMJ Open 2013;3 e003591.

24. Bartholomew LK, Markham CM, Ruiter R, et al. Planning health promotion programs: an intervention mapping approach. 4th edn San Francisco: Jossey-Bass, 2016.

25. Moore GF, Audrey S, Barker M, et al. Process evaluation of complex interventions: Medical Research Council guidance. BMJ 2015;350: h1258.

26. Dillman D. Mail and other self-administered questionnaires. In: Ross $\mathrm{PH}$, Wright JD, Anderson AB, eds. Handbook of survey research. New York: Academic Press, 1983:359-77.

27. Gilleland J, Amaral S, Mee L, et al. Getting ready to leave: transition readiness in adolescent kidney transplant recipients. J Pediatr Psychol 2012;37:85-96.

28. Rönning $\mathrm{H}$, Arestedt $\mathrm{K}$, Erik Nielsen $\mathrm{N}$, et al. Development and psychometric evaluation of the knowledge scale for adults with congenitally malformed hearts. J Cardiovasc Nurs 2013;28:228-37.

29. Yang HL, Chen YC, Wang JK, et al. Measuring knowledge of patients with congenital heart disease and their parents: validity of the 'Leuven Knowledge Questionnaire for Congenital Heart Disease'. Eur J Cardiovasc Nurs 2012;11:77-84. 
30. Goossens E, Luyckx K, Mommen N, et al. Health risk behaviors in adolescents and emerging adults with congenital heart disease: psychometric properties of the Health Behavior Scale-Congenital Heart Disease. Eur J Cardiovasc Nurs 2013;12:544-57.

31. Varni JW, Burwinkle TM, Seid M, et al. The PedsQL* 4.0 as a pediatric population health measure: feasibility, reliability, and validity. Ambul Pediatr 2003:3:329-41.

32. Petersen $\mathrm{S}$, Hagglof $\mathrm{B}$, Stenlund $\mathrm{H}$, et al. Psychometric properties of the Swedish PedsQL, pediatric quality of life inventory 4.0 generic core scales. Acta Paediatr 2009;98:1504-12.

33. Bratt E-L, Luyckx K, Goossens E, et al. Patient-reported health in young people with congenital heart disease transitioning to adulthood. J Adolesc Health 2015;57:658-65.

34. Sand $P$, Kljajic $M$, Sunnegårdh $J$. The reliability of the pediatric quality of life inventory 3.0 Cardiac Module for Swedish children with congenital heart defects. Nordic Psychol 2013;65:210-23.

35. Dyer MT, Goldsmith KA, Sharples LS, et al. A review of health utilities using the EQ-5D in studies of cardiovascular disease. Health Qual Life Outcomes 2010;8:1-12.

36. Moons P, Van Deyk K, De Bleser L, et al. Quality of life and health status in adults with congenital heart disease: a direct comparison with healthy counterparts. Eur J Cardiovasc Prev Rehabil 2006;13:407-13.

37. Luyckx K, Missotten L, Goossens E, et al. Individual and contextual determinants of quality of life in adolescents with congenital heart disease. J Adolesc Health 2012;51:122-8.

38. Apers S, Moons P, Goossens E, et al. Sense of coherence and perceived physical health explain the better quality of life in adolescents with congenital heart disease. Eur J Cardiovasc Nurs 2013;12:475-83.

39. EuroQoL Group. EuroQol-a new facility for the measurement of health-related quality of life. Health Policy 1990;16:199-208.

40. Moons $\mathrm{P}$, Marquet $\mathrm{K}$, Budts W, et al. Validity, reliability and responsiveness of the "Schedule for the Evaluation of Individual Quality of Life-Direct Weighting" (SEIQoL-DW) in congenital heart disease. Health Qual Life Outcomes 2004;2:1-8.
41. Apers S, Kovacs AH, Luyckx K, et al. Assessment of Patterns of Patient-Reported Outcomes in Adults with Congenital Heart diseaseInternational Study (APPROACH-IS): rationale, design, and methods. Int J Cardiol 2015;179:334-42.

42. Hilderson D, Moons $\mathrm{P}$, Van der Elst $\mathrm{K}$, et al. The clinical impact of a brief transition programme for young people with juvenile idiopathic arthritis: results of the DON'T RETARD project. Rheumatology (Oxford) 2016;55:133-42.

43. World Medical Association. World medical association declaration of Helsinki: ethical principles for medical research involving human subjects. JAMA 2013;310:2191-4.

44. Moher D, Hopewell S, Schulz KF, et al. CONSORT 2010 explanation and elaboration: updated guidelines for reporting paralle group randomised trials. BMJ 2010;340:c869.

45. Bourbonniere MC, Russell DJ, Goldsmith $\mathrm{CH}$. Authorship issues: one research center's experience with developing author guidelines. Am J Occup Ther 2006;60:111-17.

46. Whellan DJ, Ellis SJ, Kraus WE, et al. Method for establishing authorship in a multicenter clinical trial. Ann Intern Med 2009;151:414-20.

47. Asp A, Bratt EL, Bramhagen AC. Transfer to adult careexperiences of young adults with congenital heart disease. $J$ Pediatr Nurs 2015;30:e3-10.

48. Burstrom A, Ojmyr-Joelsson M, Bratt EL, et al. Adolescents with congenital heart disease and their parents: needs before transfer to adult care. J Cardiovasc Nurs 2016;31:399-404

49. Craig P, Dieppe P, Macintyre S, et al. Developing and evaluating complex interventions: the new Medical Research Council guidance. BMJ 2008;337:a1655

50. Erlen JA, Tamres LK, Reynolds N, et al. Assessing usual care in clinical trials. West J Nurs Res 2015;37:288-98.

51. Sable C, Foster E, Uzark K, et al. Best practices in managing transition to adulthood for adolescents with congenital heart disease: the transition process and medical and psychosocial issues: a scientific statement from the American Heart Association. Circulation 2011;123:1454-85. 